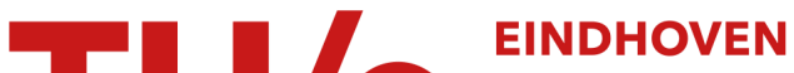 \\ UNIVERSITY OF \\ TECHNOLOGY
}

\section{The infinite volume limit of dissipative Abelian sandpiles}

Citation for published version (APA):

Maes, C., Redig, F. H. J., \& Saada, E. (2002). The infinite volume limit of dissipative Abelian sandpiles. (SPORReport : reports in statistics, probability and operations research; Vol. 200213). Technische Universiteit Eindhoven.

Document status and date:

Published: 01/01/2002

\section{Document Version:}

Publisher's PDF, also known as Version of Record (includes final page, issue and volume numbers)

\section{Please check the document version of this publication:}

- A submitted manuscript is the version of the article upon submission and before peer-review. There can be important differences between the submitted version and the official published version of record. People interested in the research are advised to contact the author for the final version of the publication, or visit the $\mathrm{DOI}$ to the publisher's website.

- The final author version and the galley proof are versions of the publication after peer review.

- The final published version features the final layout of the paper including the volume, issue and page numbers.

Link to publication

\section{General rights}

Copyright and moral rights for the publications made accessible in the public portal are retained by the authors and/or other copyright owners and it is a condition of accessing publications that users recognise and abide by the legal requirements associated with these rights.

- Users may download and print one copy of any publication from the public portal for the purpose of private study or research.

- You may not further distribute the material or use it for any profit-making activity or commercial gain

- You may freely distribute the URL identifying the publication in the public portal.

If the publication is distributed under the terms of Article 25fa of the Dutch Copyright Act, indicated by the "Taverne" license above, please follow below link for the End User Agreement:

www.tue.nl/taverne

Take down policy

If you believe that this document breaches copyright please contact us at:

openaccess@tue.nl

providing details and we will investigate your claim. 
SPOR-Report 2002-13

The infinite volume limit of dissipative Abelian sandpiles
C. Maes
F. Redig
E. Saada

SPOR-Report

Reports in Statistics, Probability and Operations Research

Eindhoven, September 2002

The Netherlands 


\title{
The Infinite Volume Limit of Dissipative Abelian Sandpiles
}

\author{
C. Maes* \\ F. Redig ${ }^{\dagger}$ \\ E. Saada $\stackrel{\ddagger}{\ddagger}$
}

12th September 2002

\begin{abstract}
We construct the thermodynamic limit of the stationary measures of the Bak-Tang-Wiesenfeld sandpile model with a dissipative toppling matrix (sand grains may disappear at each toppling). We prove uniqueness and mixing properties of this measure and we obtain an infinite volume ergodic Markov process leaving it invariant. We show how to extend the Dhar formalism of the 'abelian group of toppling operators' to infinite volume in order to obtain a compact abelian group with a unique Haar measure representing the uniform distribution over the recurrent configurations that create finite avalanches ${ }^{1}$.
\end{abstract}

\section{Introduction}

The abelian sandpile is a model on a lattice in which a discrete height-variable (e.g. representing the slope of a sandpile at that site) is associated to each lattice site. A configuration of height variables is called stable if the height at any site is less than a critical value $\gamma$, otherwise it is called unstable. The mechanism of stabilization of an unstable configuration is by a sequence of so-called topplings. In such a toppling, the site gives one (sand) grain to each of its neighboring sites which in their turn can become "unstable" and "topple" etc., until every site has again a subcritical heightvalue. An unstable site thus creates an "avalanche" involving possibly the toppling of many sites around it. The dynamics on stable configurations consists in adding at

${ }^{*}$ Instituut voor Theoretische Fysica, Celestijnenlaan 200D, 3001 Heverlee, Belgium

${ }^{\dagger}$ Faculteit Wiskunde en Informatica, Technische Universiteit Eindhoven, Postbus 513, 5600 MB Eindhoven, The Netherlands

${ }^{\ddagger}$ CNRS, UMR 6085, Laboratoire de mathématiques Raphaël Salem, Université de Rouen, site Colbert, 76821 Mont-Saint-Aignan Cedex, France

${ }^{1}$ MSC 2000: Primary-82C22; secondary-60K35.

Key-words: Sandpile dynamics, Nonlocal interactions, Interacting particle systems, Thermodynamic limit, Dissipative systems, Decay of correlations and Mass-gap. 
randomly chosen sites and stabilizing the configuration. Since the avalanche of topplings can involve many sites (depending on the configuration) this dynamics is highly nonlocal. Therefore, defining infinite volume limits poses a non-trivial problem.

Since its appearance in [1], the abelian sandpile model has been studied intensively, see for instance [4], [5], [8], [14], and also [12] for a mathematical review of the main properties of the model in finite volume. The main technical tool in the analysis of the model is the "abelian group" of toppling operators which can be identified with the set of recurrent configurations.

Our aim is to define the model on infinite graphs. The main problem to overcome is the non-locality or extreme sensitivity to boundary-conditions of the dynamics. We have constructed in [9] and [10] the infinite volume standard sandpile process on the one-dimensional lattice and on homogeneous trees. In the present paper we focus on the thermodynamic limit for dissipative models. There, the infinite graph $S$ is a subgraph of the regular lattice $\mathbb{Z}^{d}$ and on each site the height has a critical value $\gamma$, an integer not less than the maximal number $\mathcal{N}$ of neighbors of a site in $S$. The finite volume rule now starts as follows: choose a site $x$ at random from the volume $V$ and add one grain to it. Suppose that $x$ has $\mathcal{N}_{x}$ nearest neighbors and that the new height at $x$ is $\gamma+1$. Then, it topples by giving to each of its nearest neighbors one grain and dissipating $\gamma-\mathcal{N}_{x}$ grains to a sink associated to the volume. We say that the site $x$ is dissipative when $\gamma>\mathcal{N}_{x}$ and the model is dissipative when this happens for a considerable fraction of sites. This condition can be rephrased in terms of the simple random walk on $S$ with a sink associated to the dissipative sites: the model is dissipative when the Green's function decays fast enough in the lattice distance, see (2.12) below for a precise formulation. Dissipative abelian sandpile models have appeared in the physics literature in [15] and [3]; where it was argued that dissipation removes criticality, i.e., correlation functions decay exponentially fast uniformly in the volume. From the point of view of defining the thermodynamic limit, the main simplification of dissipative models is that there is a stronger control of the non-locality: more precisely, the probability that a site $y$ is influenced by addition on $x$ decays exponentially fast (or at least in a summable way) in the distance between the sites. Hence avalanche clusters are almost surely finite.

\section{$1.1 \quad$ Results}

Our three main results are:

1. The extension of the Dhar formalism to infinite volume sandpile dynamics. That includes the construction of a compact abelian group of recurrent configurations on which we can define addition (of sand) operations.

2. The construction of the thermodynamic limit of the finite volume stationary measure with exponential decay of correlations in the case of "strong dissipativity".

3. The construction of an infinite volume sandpile process which converges exponentially fast to its unique stationary measure. 


\subsection{Plan of the paper}

The paper is organized as follows: in Section 2 we repeat some of the basic results on the abelian sandpile model in finite volume and we introduce the definition of dissipativity, with examples. In Section 3 we show how to extend the dynamics on infinite volume recurrent configurations and we recover the group structure of "addition of recurrent configurations." In Section 4 we prove existence and ergodic properties of the infinite volume dynamics. Section 5 is devoted to the proof of exponential decay of correlations.

\section{Finite volume model}

In this section we recall some definitions and properties of abelian sandpiles in finite volume. In [4], [5], [8], [14] and [12], the reader will find more details.

The infinite graphs $S$ on which we construct the dissipative abelian sandpile dynamics are $S=\mathbb{Z}^{d}$, and "strips", i.e., $S=\mathbb{Z} \times\{1, \ldots, \ell\}$, for some integer $\ell>1$ (notice that $\ell=1$ corresponds to $S=\mathbb{Z}^{d}$ with $d=1$ ). Finite subsets of $S$ will be denoted by $V, W$; we write $\mathcal{S}=\{W \subset S: W$ finite $\}$. We denote by $\partial V$ the external boundary of $V$ : all the sites in $S \backslash V$ that have a nearest neighbor in $V$. Let $\mathcal{N}$ be the maximal number of neighbors of a site in $S$, e.g., $\mathcal{N}=2 d$ for $S=\mathbb{Z}^{d}$ and $\mathcal{N}=4$ for $S=\mathbb{Z} \times\{1, \ldots, \ell\}, \ell \geq 3$. The state space of the process in infinite volume is $\Omega=\{1, \ldots, \gamma\}^{S}$, with some integer $\gamma \geq \mathcal{N}$

We fix $V \in \mathcal{S}$, a nearest neighbor connected subset of $S$. Then $\Omega_{V}=\{1, \ldots, \gamma\}^{V}$ is the state space of the process in the finite volume $V$. We denote by $\mathcal{N}_{V}(x)$ the number of nearest neighbors of $x$ in $V$.

A (infinite volume) height configuration $\eta$ is a mapping from $S$ to $\mathbb{N}=\{1,2, \ldots\}$ assigning to each site $x$ a "number of sand grains" $\eta(x) \geq 1$. If $\eta \in \Omega$, it is called a stable configuration. Otherwise $\eta$ is unstable. For $\eta \in \Omega, \eta_{V}$ is its restriction to $V$, and for $\eta, \zeta \in \Omega, \eta_{V} \zeta_{V^{c}}$ denotes the configuration whose restriction to $V$ (resp. $V^{c}$ ) coincides with $\eta_{V}$ (resp. $\zeta_{V c}$ ).

The configuration space $\Omega$ is endowed with the product topology, making it into a compact metric space. A function $f: \Omega \rightarrow \mathbb{R}$ is local if there is a finite $W \subset S$ such that $\eta_{W}=\zeta_{W}$ implies $f(\eta)=f(\zeta)$. The minimal (in the sense of set ordering) such $W$ is called the dependence set of $f$, and is denoted by $D_{f}$. A local function can be seen as a function on $\Omega_{W}$ for all $W \supset D_{f}$, and every function on $\Omega_{W}$ can be seen as a local function on $\Omega$. The set $\mathcal{L}$ of all local functions is uniformly dense in the set $\mathcal{C}(\Omega)$ of all continuous functions on $\Omega$.

\subsection{The dynamics in finite volume}

The toppling matrix $\Delta$ on $S$ is defined by, for $x, y \in S$,

$$
\begin{aligned}
& \Delta_{x x}=\gamma \\
& \Delta_{x y}=-1 \text { if } x \text { and } y \text { are nearest neighbors, } \\
& \Delta_{x y}=0 \text { otherwise }
\end{aligned}
$$


We denote by $\Delta^{V}$ the restriction of $\Delta$ to $V \times V$.

A site $x \in V$ is called a dissipative site in the volume $V$ if

$$
\sum_{y \in V} \Delta_{x y}>0 .
$$

Thus if $\gamma>\mathcal{N}$, every site is dissipative. If $\gamma=\mathcal{N}$, the internal boundary sites of $V$ (i.e. all the sites in $V$ that have a nearest neighbor in $S \backslash V$ ), are the only dissipative sites in $V$. To define the sandpile dynamics, we first introduce the toppling of a site $x$ as the mapping $T_{x}: \mathbb{N}^{V} \rightarrow \mathbb{N}^{V}$ defined by

$$
\begin{aligned}
T_{x}(\eta)(y) & =\eta(y)-\Delta_{x y} \text { if } \eta(x)>\Delta_{x x} \\
& =\eta(y) \text { otherwise. }
\end{aligned}
$$

In words, site $x$ topples if and only if its height is strictly larger than $\Delta_{x x}=\gamma$, by transferring $-\Delta_{x y} \in\{0,1\}$ grains to site $y \neq x$ and losing itself in total $\Delta_{x x}=\gamma$ grains. As a consequence, if the site is dissipative, then, upon toppling, some grains are lost. Toppling rules commute on unstable configurations, i.e., for $x, y \in S$ such that $\eta(x)>$ $\gamma=\Delta_{x x}$ and $\eta(y)>\gamma=\Delta_{y y}$ :

$$
T_{x}\left(T_{y}(\eta)\right)=T_{y}\left(T_{x}(\eta)\right)
$$

For $\eta \in \mathbb{N}^{V}$, we say that $\zeta \in \Omega_{V}$ arises from $\eta$ by toppling if there exists a $k$-tuple $\left(x_{1}, \ldots, x_{k}\right)$ of sites in $V$ such that

$$
\zeta=\left(\prod_{i=1}^{k} T_{x_{i}}\right)(\eta)
$$

The toppling transformation is the mapping $\mathcal{T}: \mathbb{N}^{V} \rightarrow \Omega_{V}$ defined by the requirement that $\mathcal{T}(\eta)$ arises from $\eta$ by toppling. The fact that stabilization of an unstable configuration is always possible follows from the existence of dissipative sites. The fact that $\mathcal{T}$ is well-defined, i.e., that the same final stable configuration is obtained irrespective of the order of the topplings, is a consequence of the commutation property, see [12] for a complete proof.

For $\eta \in \mathbb{N}^{V}$ and $x \in V$, let $\eta^{x}$ denote the configuration obtained from $\eta$ by adding one grain to site $x$, i.e. $\eta^{x}(y)=\eta(y)+\delta_{x, y}$. The addition operator defined by

$$
a_{x, V}: \Omega_{V} \rightarrow \Omega_{V} ; \eta \mapsto a_{x, V} \eta=\mathcal{T}\left(\eta^{x}\right)
$$

represents the effect of adding a grain to the stable configuration $\eta$ and letting a stable configuration arise by toppling. Because $\mathcal{T}$ is well-defined, the composition of addition operators is commutative. We can now define a discrete time Markov chain $\left\{\eta_{n}: n \geq 0\right\}$ on $\Omega_{V}$ by picking a point $x \in V$ randomly at each discrete time step and applying the addition operator $a_{x, V}$ to the configuration. We define also a continuous time Markov process $\left\{\eta_{t}: t \geq 0\right\}$ with infinitesimal generator

$$
L_{V}^{0, \varphi} f(\eta)=\sum_{x \in V} \varphi(x)\left[f\left(a_{x, V} \eta\right)-f(\eta)\right]
$$

this is a pure jump process on $\Omega_{V}$, where $\varphi: S \rightarrow(0, \infty)$ is the addition rate function. 


\subsection{Recurrent configurations, invariant measure}

The Markov chain $\left\{\eta_{n}, n \geq 0\right\}$ (or its continuous time version $\left\{\eta_{t}\right\}$ ) has a unique recurrent class $\mathcal{R}_{V}$, and its stationary measure $\mu_{V}$ is the uniform measure on that class, i.e.,

$$
\mu_{V}=\frac{1}{\left|\mathcal{R}_{V}\right|} \sum_{\eta \in \mathcal{R}_{V}} \delta_{\eta}
$$

A configuration $\eta \in \Omega_{V}$ belongs to $\mathcal{R}_{V}$ if it passes the burning algorithm (see [4]), which is described as follows. Pick $\eta \in \Omega_{V}$ and erase the set $E_{1}$ of all sites $x \in V$ with a height strictly larger than the number of neighbors of that site in $V$, i.e., satisfying the inequality

$$
\eta(x)>\mathcal{N}_{V}(x)
$$

Iterate this procedure for the new volume $V \backslash E_{1}$, and the new matrix $\Delta^{V \backslash E_{1}}$ and so on. If at the end some non-empty subset $V_{f}$ is left, $\eta$ satisfies, for all $x \in V_{f}$,

$$
\eta(x) \leq \mathcal{N}_{V_{f}}(x)
$$

The restriction $\eta_{V_{f}}$ is then called a forbidden subconfiguration. If $V_{f}$ is empty, the configuration is called allowed. The set $\mathcal{A}_{V}$ of allowed configurations coincides with the set of recurrent configurations, $\mathcal{A}_{V}=\mathcal{R}_{V}$ (see [8], [12], [14]).

A recurrent configuration is thus nothing but a configuration without forbidden subconfigurations. This easily extends to infinite volume:

Definition 2.6 A configuration $\eta \in \Omega$ is called recurrent if for any $V \in \mathcal{S}, \eta_{V} \in \mathcal{R}_{V}$.

The set $\mathcal{R}$ of all recurrent configurations forms a perfect (hence uncountable) subset of $\Omega$. This means that $\mathcal{R}$ is closed (hence compact) and every element $\eta \in \mathcal{R}$ is the limit of a sequence $\eta_{n} \in \mathcal{R}, \eta_{n} \neq \eta$.

On the set $\mathcal{R}_{V}$, the finite volume addition operators $a_{x, V}$ can be inverted and they generate a finite abelian group. This group is characterized by the closure relation

$$
\prod_{y \in V} a_{y, V}^{\Delta_{x y}^{V}}=\mathrm{Id}
$$

By the group property, the uniform measure $\mu_{V}$ is invariant under the action of $a_{x, V}$ and of $a_{x, V^{*}}^{-1}$.

\subsection{Toppling numbers}

For $x, y \in V$ and $\eta \in \Omega_{V}$, let $n_{V}(x, y, \eta)$ denote the number of topplings at site $y \in V$ by adding a grain at $x \in V$, i.e., the number of times we have to apply the operator $T_{y}$ to stabilize $\eta+\delta_{x}$ in the volume $V$. We have the relation

$$
\eta(y)+\delta_{x, y}=a_{x, V} \eta(y)+\sum_{z \in V} \Delta_{y z}^{V} n_{V}(x, z, \eta)
$$


Defining

$$
G_{V}(x, y)=\int \mu_{V}(d \eta) n_{V}(x, y, \eta)
$$

one obtains, by integrating (2.8) over $\mu_{V}$ :

$$
G_{V}(x, y)=\left(\Delta^{V}\right)_{x y}^{-1}
$$

In the limit $V \uparrow S, G_{V}$ converges to the Green's function $G$ of the simple random walk on $S$ with a sink associated to the dissipative sites (i.e. every site is linked with $\gamma-\mathcal{N}_{S}$ edges to a sink and the walk stops when it reaches the sink). By (2.9), the probability that a site $y$ topples by addition at $x$ in volume $V$ is bounded by $G_{V}(x, y)$.

Definition 2.11 We say that the sandpile model is dissipative if

$$
\sup _{x \in S} \sum_{y \in S} G(x, y)<+\infty
$$

In our examples, if $\gamma>2 d$ for $\mathbb{Z}^{d}$ or $\gamma \geq 4$ for strips, the Green's function $G(x, y)$ decays exponentially in the lattice distance between $x$ and $y$ and hence (2.1) defines a dissipative model. From now on, we restrict ourselves to these cases.

Definition 2.13 For any integer $n$, let $\nu_{W_{n}}$ be a probability measure on $\Omega_{W_{n}}$, with $W_{n} \in \mathcal{S}, W_{n} \uparrow S$. Then $\nu_{W_{n}}$ converges to a probability measure $\nu$ on $\Omega$ if for any $f \in \mathcal{L}$,

$$
\lim _{n \rightarrow \infty} \int f d \nu_{W_{n}}=\int f d \nu .
$$

We denote by $\mathcal{I}$ the set of all limit points of $\left\{\mu_{V}: V \in \mathcal{S}\right\}$ in the sense of Definition 2.13. By compactness of $\Omega, \mathcal{I}$ is a non-empty compact convex set. Moreover, by (2.5) and Definition 2.6, any $\mu \in \mathcal{I}$ concentrates on $\mathcal{R}$ (see [10]).

\subsection{Untoppling numbers}

On the set $\mathcal{R}_{V}$ the addition operators $a_{x}$ are invertible. The action of the inverse on a recurrent configuration can be defined recursively as follows, see [8]. Consider $\eta \in \mathcal{R}_{V}$ and $x \in V$. Remove one grain from $\eta$ at site $x$. If the resulting configuration is recurrent, it is $a_{x, V}^{-1} \eta$, otherwise it contains a forbidden subconfiguration (fsc) in $V_{1} \subset V$. In that case "untopple" the sites in $V_{1}$. By untoppling of a site $z$ we mean that the sites are updated according to the rule $\eta(y) \rightarrow \eta(y)+\Delta_{z y}$. Iterate this procedure until a recurrent configuration is obtained: the resulting recurrent configuration coincides with $a_{x, V}^{-1} \eta$

As an example, consider a graph with just three sites $a \sim b \sim c$ for $\gamma=2$ The configuration 212 is recurrent. After removal of one grain at site $c$, we get 211 , which contains the fsc 11 . Untoppling site $b$ gives 130 , and untoppling site $c$ gives 122 , which is recurrent. One easily verifies that addition at site $c$ on 122 gives back the original 
212.

Call $n_{V}^{-}(x, y, \eta)$ the number of untopplings at site $y$ by removing one grain from $x$ and from untoppling sites until a recurrent configuration is obtained. As in the previous section, one easily proves the relation

$$
\int n_{V}^{-}(x, y, \eta) \mu_{V}(d \eta)=G_{V}(x, y)
$$

\section{The group of addition operators in infinite volume}

In this section we show how to obtain the group of addition operators in the infinite volume limit. The assumption of dissipativity is crucial in order to obtain a compact abelian group in the thermodynamic limit.

\subsection{Addition operator}

The finite volume addition operators $a_{x, V}$ (cf. (2.3)) are defined on $\Omega$ via

$$
a_{x, V}: \Omega \rightarrow \Omega: \eta \mapsto a_{x, V} \eta=\left(a_{x, V} \eta_{V}\right)_{V} \eta_{V^{c}}
$$

(with some slight abuse of notation). Similarly, the inverses are defined on $\mathcal{R}$ via

$$
a_{x, V}^{-1}: \mathcal{R} \rightarrow \Omega: \eta \mapsto\left(a_{x, V}^{-1} \eta_{V}\right) \eta_{V c}
$$

Remark that if $\eta \in \mathcal{R}$, then $\left(a_{x, V} \eta\right)_{W} \in \mathcal{R}_{W}$ for all $W \subset V$ but $a_{x, V} \eta$ is not necessarily an element of $\mathcal{R}$.

Definition 3.3 For $\eta \in \Omega$, we say that the limit of the finite volume addition operators is defined on $\eta$ if for every $x \in S$, there exists $\Lambda_{0} \in \mathcal{S}$ such that for any $\Lambda \in \mathcal{S}, \Lambda \supset \Lambda_{0}$, $a_{x, \Lambda} \eta=a_{x, \Lambda_{0}} \eta$; in that case, we write

$$
a_{x} \eta=a_{x, \Lambda_{0}} \eta
$$

Similarly, for $\eta \in \mathcal{R}$, we say that the limit of the finite volume inverse addition operators is defined on $\eta$ if if for every $x \in S$, there exists $\Lambda_{0} \in \mathcal{S}$ such that for any $\Lambda \in \mathcal{S}, \Lambda \supset \Lambda_{0}$, $a_{x, \Lambda}^{-1} \eta=a_{x, \Lambda_{0}}^{-1} \eta ;$ in that case, we write

$$
a_{x}^{-1} \eta=a_{x, \Lambda_{0}}^{-1} \eta
$$

Remark that if $\eta \in \mathcal{R}$ and $a_{x}$ is defined on $\eta$, then $a_{x} \eta \in \mathcal{R}$.

Lemma 3.4 Assume (2.12). For any $\mu \in \mathcal{I}$ there exists a tail measurable subset $\bar{\Omega} \subset \Omega$ such that:

1. $\mu(\bar{\Omega})=1$; 
2. The limit of the finite volume addition operators and their inverses is defined on every $\eta \in \bar{\Omega}$.

Moreover, every $\mu \in \mathcal{I}$ is invariant under the action of $a_{x}$, i.e., for all $x \in S$ and $f \in \mathcal{L}$

$$
\int f\left(a_{x} \eta\right) \mu(d \eta)=\int f(\eta) \mu(d \eta)
$$

Proof. We prove the result for the addition operators, the analogue for the inverses is proved along the same lines by replacing "number of topplings" by "number of untopplings".

Pick $W_{k} \in \mathcal{S}, W_{k} \uparrow S$ such that $\mu_{W_{k}} \rightarrow \mu$ and $x \in S$. We have to prove that

$$
\mu\left[\forall \Lambda_{0} \in \mathcal{S}, \exists V \supset \Lambda_{0}: a_{x, V} \eta \neq a_{x, \Lambda_{0}} \eta\right]=0
$$

We enumerate $S=\left\{x_{n}: n \in \mathbb{N}\right\}$, with $V_{n}=\left\{x_{1}, \ldots, x_{n}\right\}$ such that $V_{n} \uparrow S, x_{n} \in \partial V_{n-1}$. If $a_{x, V} \eta \neq a_{x, V_{n}} \eta$, then some boundary site of $V_{n}$ has toppled under addition at $x$ in volume $V$. This implies that for every $m$ such that $V_{m} \supset V$ some external boundary site of $V_{n}$ topples upon addition at $x$ in $V_{m}$. Therefore, the left hand side of (3.6) is bounded by

$$
\mu\left[\forall n \in \mathbb{N}, \exists p \geq n, \exists y \in \partial V_{n}: n_{V_{p}}(x, y, \eta) \geq 1\right]
$$

and we have to estimate

$$
\mu\left[\exists p \geq n, \exists y \in \partial V_{n}: n_{V_{p}}(x, y, \eta) \geq l\right]
$$

Since $n_{V_{p}}(x, y, \eta) \leq n_{V_{p+1}}(x, y, \eta)$,

$$
\begin{aligned}
\mu\left(\exists p \geq n, \exists y \in \partial V_{n}: n_{V_{p}}(x, y, \eta) \geq 1\right) & \leq \lim _{k \rightarrow \infty} \mu\left(\exists y \in \partial V_{n}: n_{V_{k}}(x, y, \eta) \geq 1\right) \\
& \leq \lim _{k \rightarrow \infty} \sum_{y \in \partial V_{n}} \int n_{V_{k}}(x, y, \eta) \mu(d \eta) \\
& \leq \lim _{k \rightarrow \infty} \sum_{y \in \partial V_{n}} \int n_{W_{k}}(x, y, \eta) \mu_{W_{k}}(d \eta) \\
& =\sum_{y \in \partial V_{n}} G(x, y)
\end{aligned}
$$

which implies that (3.7) converges to zero as $n$ tends to infinity, by condition (2.12). Finally, (3.5) follows easily from Definition $3.3, \mu(\bar{\Omega})=1, f \in \mathcal{L}$, and the invariance of $\mu_{V}$ under the finite volume addition operator $a_{x, V}$.

Notice that for $\eta \in \bar{\Omega}$, we can take the limit $V \uparrow S$ in (2.8) and write

$$
\eta(y)+\delta_{x, y}=a_{x} \eta(y)+\sum_{z \in S} \Delta_{y z} n_{S}(x, z, \eta)
$$

for any $x, y \in S$, where $n_{S}(x, z, \eta)$, the number of topplings at site $z \in S$ by adding a grain at $x$, satisfies $\sum_{z \in S} n_{S}(x, z, \eta)<+\infty$. 
Lemma 3.9 Assume (2.12). For any $\mu \in \mathcal{I}$ there exists a tail measurable subset $\Omega^{\circ} \subset$ $\vec{\Omega}$ with $\mu\left(\Omega^{\circ}\right)=1$ such that for any $V \in \mathcal{S}$ and $n_{x}, x \in V$ integers, the product $\prod_{x \in V} a_{x}^{n_{x}}$ is well-defined, as the limit of $\prod_{x \in V} a_{x, \Lambda}^{n_{x}}$ as $\Lambda \rightarrow S$, on every $\eta \in \Omega^{\circ}$.

Proof. We fix $x \in V, n_{x}$ a positive integer, and we prove that $a_{x}^{n_{x}}$ is well-defined on $\bar{\Omega}$ (the case of negative $n_{x}$ is similar and the extension to finite products it straightforward). Following the same lines as in the preceding proof, we have to replace (3.6) by

$$
\mu\left[\forall \Lambda_{0} \in \mathcal{S}, \exists V \supset \Lambda_{0}: a_{x, V}^{n_{x}} \eta \neq a_{x, \Lambda_{0}}^{n_{x}} \eta\right]=0
$$

We denote by $E_{V_{p}}\left(n_{z}, z, x, \eta\right)$ the event that addition in $V_{p}$ of $n_{z}$ grains at $z$ causes at least one toppling at $x$. As these events are increasing in $p$, we estimate

$$
\begin{aligned}
\mu\left(\exists p \geq n, \exists y \in \partial V_{n}: E_{V_{p}}\left(n_{x}, x, y, \eta\right) \geq 1\right) & \leq \lim _{k \rightarrow \infty} \sum_{y \in \partial V_{n}} \mu_{W_{k}}\left(E_{W_{k}}\left(n_{x}, x, y, \eta\right)\right) \\
& \leq \sum_{y \in \partial V_{n}} n_{x} G(x, y)
\end{aligned}
$$

where the last inequality is a consequence of (2.10) and (3.5). From this we easily obtain that for any $V \in \mathcal{S}, n \in \mathbb{Z}^{V}$, the product $\prod_{x \in V} a_{x}^{n_{x}}$ is well-defined on a tail measurable set $\Omega(V, n)$ of $\mu$-measure one. The set $\Omega^{\circ}$ is then the countable intersection

$$
\Omega^{o}=\cap_{V \in \mathcal{S}, n \in \mathbb{Z}^{V}} \Omega(V, n)
$$

of tail measurable $\mu$-measure one sets.

The following proposition extends this to addition on infinite products.

Proposition 3.10 Assume (2.12). If $n=\left(n_{x}, x \in S\right) \in \mathbb{Z}^{S}$ satisfies $\sum_{x \in S}\left|n_{x}\right| G(0, x)<$ $+\infty$, the product $\prod_{x \in S} a_{x}^{n_{x}}$ is well-defined on a set $\Omega(n)$ of $\mu$-measure 1 , for every $\mu \in \mathcal{I}$.

Proof. Take $n_{x} \geq 0$; the case of negative $n_{x}$ is treated again by replacing "topplings" with "untopplings". It suffices to show that for every $\Lambda_{0} \in \mathcal{S}$

$$
\mu\left(\exists V_{0}, \forall V \supset V_{0}, \forall y \in \Lambda_{0}:\left(\prod_{x \in V} a_{x}^{n_{x}} \eta\right)(y)=\left(\prod_{x \in V_{0}} a_{x}^{n_{x}} \eta\right)(y)\right)=1
$$

or

$$
\lim _{V_{0} \uparrow S} \mu\left(\exists V \supset V_{0}, \exists y \in \Lambda_{0}:\left(\prod_{x \in V} a_{x}^{n_{x}} \eta\right)(y) \neq\left(\prod_{x \in V_{0}} a_{x}^{n_{x}} \eta\right)(y)\right)=0
$$

The left hand side of (3.11) is bounded by the sum

$$
\sum_{y \in \Lambda_{0}} \mu\left(\exists V \supset V_{0}:\left(\prod_{x \in V} a_{x}^{n_{x}} \eta\right)(y) \neq\left(\prod_{x \in V_{0}} a_{x}^{n_{x}} \eta\right)(y)\right)
$$


If none of the external boundary points of $\Lambda_{0}$ topples upon addition of $n_{z}$ grains at $z \in V \backslash V_{0}$ to the configuration $\left(\prod_{x \in V_{0}} a_{x}^{n_{x}} \eta\right)$, we have that for all $y \in \Lambda_{0}$ :

$$
\left(\prod_{x \in V} a_{x}^{n_{x}} \eta\right)(y)=\left(\prod_{x \in V_{0}} a_{x}^{n_{x}} \eta\right)(y)
$$

Since $\mu$ is invariant under the $a_{x}$, see (3.5), the sum (3.12) is bounded from above by

$$
\sum_{y \in \Lambda_{0}} \sum_{|x-y|=1} \sum_{z \in V_{0}^{c}} \mu\left(E_{S}\left(n_{z}, z, x, \eta\right)\right) \leq \sum_{y \in \Lambda_{0}} \sum_{|x-y|=1} \sum_{z \in V_{0}^{c}} n_{z} G(z, x)
$$

which implies (3.11) by the hypothesis on $n$.

\subsection{Group structure}

Here we show that the product $\prod_{x \in S} a_{x}^{n_{x}}$ can be defined on any recurrent configuration, provided we identify recurrent configurations which differ by a multiple of $\Delta$.

Given $n \in \mathbb{Z}^{S}$ and $\eta \in \mathcal{R}$, we consider the set

$$
A_{n}(\eta)=\left\{\xi \in \mathcal{R}: \exists m \in \mathbb{Z}^{S}, \eta+n=\xi+\Delta m\right\}
$$

Similarly, for subtraction,

$$
S_{n}(\eta)=\left\{\xi \in \mathcal{R}: \exists m \in \mathbb{Z}^{S}, \eta-n=\xi+\Delta m\right\}
$$

Fix $n \in \mathbb{Z}^{S}$ so that

$$
\sup _{y \in S} \sum_{x \in S}\left[\left|n_{x}\right|+2 \gamma\right] G(y, x)=B<+\infty
$$

and let

$$
\Omega_{n}=\left\{\eta \in \mathcal{R}: S_{n}(\eta) \neq \emptyset, A_{n}(\eta) \neq \emptyset\right\}
$$

be the set of recurrent configurations for which both addition and substraction with $n$ gives rise to a new recurrent configuration, modulo the toppling matrix applied to an integer function.

Lemma $3.14 \Omega_{n}=\mathcal{R}$.

Proof. We prove that $\Omega_{n}$ is closed. Let $\left(\eta_{k}\right)_{k \geq 0}$ be a sequence in $\Omega_{n}$ which converges to $\eta$ as $k \rightarrow \infty$. For each $k$, there exist $\eta_{k}^{ \pm} \in \mathcal{R}$ and $m_{k}^{ \pm} \in[-B, B]^{S}$ such that

$$
\eta_{k} \pm n=\eta_{k}^{ \pm}+\Delta m_{k}^{ \pm}
$$

Since $\mathcal{R} \times[-B, B]^{S}$ is compact, there exists a subsequence $k_{i} \rightarrow \infty$ such that $\eta_{k_{i}}^{ \pm} \rightarrow \eta^{ \pm}$ and $m_{k_{i}}^{ \pm} \rightarrow m^{ \pm}$. Taking limits along this subsequence in (3.15) yields

$$
\eta \pm n=\eta^{ \pm}+\Delta m^{ \pm}
$$

i.e., $\eta \in \Omega_{n}$. Looking back at Lemma 3.10, $\Omega(n) \cap \mathcal{R} \subset \Omega_{n}$ and $\Omega(n)$ is a $\mu$-measure one (hence non-empty) tail set. Therefore it is dense and $\Omega_{n}=\mathcal{R}$. 
Definition 3.16 Two recurrent configurations $\eta, \zeta \in \mathcal{R}$ are called equivalent, and we write $\eta \sim \zeta$, if there exists $m \in \mathbb{Z}^{S}$ such that

$$
\eta=\zeta+\Delta m
$$

Remark 3.18 1. For all $n \in \mathbb{Z}^{S}, \eta \in \mathcal{R}$, if $\zeta, \zeta^{\prime} \in A_{n}(\eta)$ (or $\zeta, \zeta^{\prime} \in S_{n}(\eta)$ ), then $\zeta \sim \zeta^{\prime}$

2. If $\eta \sim \eta^{\prime}$, then $A_{n}(\eta)=A_{n}\left(\eta^{\prime}\right), S_{n}(\eta)=S_{n}\left(\eta^{\prime}\right)$ for all $n \in \mathbb{Z}^{S}$.

3. In the finite volume case one can prove that every equivalence class in $\mathbb{Z}^{V} / \Delta^{V} \mathbb{Z}^{V}$ contains exactly one recurrent configuration, i.e., $\eta, \zeta \in \mathcal{R}_{V}$ and

$$
\eta=\zeta+\Delta^{V} m
$$

imply $\eta=\zeta$. This is no longer true in infinite volume. As an example we take $S=\mathbb{Z} \times\{1,2\}, \gamma=4$. Then the recurrent configurations $\eta(x)=3$ for all $x$ and $\zeta(x)=4$ for all $x$ (denoted by $\overline{3}$ and $\overline{4}$ ) are equivalent:

$$
\zeta=\eta+\Delta m
$$

where $m(x)=1$ for all $x$.

We can now introduce the addition operator on classes: take the class $[\eta]$ containing the recurrent configuration $\eta$, let $\xi \in A_{n}(\eta)$ and define

$$
\prod_{x \in S} a_{x}^{n_{x}}[\eta]=[\xi]
$$

Notice that if $\eta \in \bar{\Omega}$ (i.e., $\eta \in \mathcal{R}$ is such that $a_{x}$ is the limit of $a_{x, V}$ on $\eta$ ), then

$$
a_{x}[\eta]=\left[a_{x} \eta\right]
$$

Proposition 3.20 Assume (2.12). $\mathcal{R} / \sim$ is a compact metric space.

Proof. It suffices to show that equivalence classes are closed. Suppose we have sequences $\left(\eta_{k}\right),\left(\xi_{k}\right)$ of recurrent configurations with $\eta_{k} \sim \xi_{k}, \eta_{k} \rightarrow \eta, \xi_{k} \rightarrow \xi$. Then, there exist $m_{k} \in[-M, M]^{S}$ with $M=2 \gamma \sup _{x \in S} \sum_{y} G(x, y)$ such that

$$
\eta_{k}=\xi_{k}+\Delta m_{k}
$$

We can choose a subsequence $k_{i} \rightarrow+\infty$ such that $m_{k_{i}} \rightarrow m$. Taking limits along this subsequence in (3.21) yields

$$
\eta=\xi+\Delta m
$$

giving $\eta \sim \xi$.

By point 2 of Remark 3.18 the addition of equivalence classes of configurations in $\mathcal{R}$ is well-defined. 
Definition 3.22 Assume (2.12). For $[\eta],[\xi]$ in $\mathcal{R} / \sim$ we define

$$
[\eta] \oplus[\xi]
$$

to be the class which contains $A_{\xi}(\eta)$.

Theorem $3.23(\mathcal{R} / \sim, \oplus)$ is a compact abelian group, hence it admits a unique Haar measure.

Proof. The group property is immediate; the compactness follows from Proposition 3.20. For the consequence see e.g. [7] p. 31.

The next result shows that from a measure theoretic perspective, there is no difference between classes of the relation $\sim$ and recurrent configurations. As a corollary, we obtain that the set $\mathcal{I}$ of possible weak limit points of the finite volume stationary measures is a singleton.

Proposition 3.24 For any $\mu \in \mathcal{I}$ there exists a set $\Omega_{\mu} \subset \mathcal{R}$ of $\mu$-measure one such that for every $\eta \in \Omega_{\mu},[\eta] \cap \Omega_{\mu}=\{\eta\}$.

Before proving the proposition, we state and prove

Theorem 3.25 The set $\mathcal{I}$ is a singleton.

Proof. Suppose that $\mathcal{I}$ contains two different measures $\mu, \nu$. Then there exists a measurable subset $A$ such that

$$
\mu(A) \neq \nu(A) .
$$

$\mu$ and $\nu$ are lifted to $\mathcal{R} / \sim$ via

$$
\bar{\mu}([A])=\mu\left(\cup_{\eta \in A}\{\eta\}\right)
$$

Let $\Omega_{\mu}, \Omega_{\nu}$ be the sets of proposition 3.24. Then

$$
\begin{aligned}
\vec{\mu}([A]) & =\mu\left(\cup_{\eta \in A}[\eta]\right) \\
& =\mu\left(\cup_{\eta \in A}[\eta] \cap \Omega_{\mu}\right) \\
& =\mu\left(\cup_{\eta \in A}\{\eta\} \cap \Omega_{\mu}\right) \\
& =\mu\left(A \cap \Omega_{\mu}\right) \\
& =\mu(A) .
\end{aligned}
$$

Analogously one has $\bar{\nu}([A])=\nu(A)$. Hence $\tilde{\mu}$ and $\bar{\nu}$ are different. Because $\mu$ and $\nu$ are invariant under the action of the addition operators $a_{x}$, it follows that $\bar{\mu}$ and $\bar{\nu}$ are different and invariant under the group action. This contradicts the uniqueness of the Haar measure.

To prove the proposition, let the set $\Omega_{\mu}$ consist of those configurations $\eta$ that satisfy 
1. For all $x \in S, a_{x}$ and $a_{x}^{-1}$ are well defined as limit of the corresponding finite volume operators, and $a_{x} a_{x}^{-1} \eta=a_{x}^{-1} a_{x} \eta=\eta$

2. For all $n_{0} \in \mathbb{N}$ there exists $N \in \mathbb{N}$ such that for all $p \geq N$ and for all $n \in$ $\{-B, \ldots, B\}^{\partial V_{p}}$

$$
\prod_{x \in \partial V_{p}} a_{x}^{n(x)} \eta
$$

coincides with $\eta$ on $V_{0}=V_{n_{0}}$.

That $\mu\left(\Omega_{\mu}\right)=1$ can be proven from the same kind of arguments used before to prove that $a_{x}$ and $a_{x}^{-1}$ are well defined on a set of measure one for every $\mu \in \mathcal{I}$. Moreover, $\mu\left(a_{x} \bar{\Omega}\right)=1$ by invariance. By the closure relation for the infinite volume addition operators, see $(2.7)$,

$$
a_{x}^{\Delta_{x x}}=\prod_{y \sim x} a_{y}^{-\Delta_{x y}}
$$

(product over nearest neighbors), we get for every $n$

$$
\prod_{x \in V_{n}} a_{x}^{\Delta m(x)}(\eta)=\prod_{x \in \partial V_{n}} a_{x}^{\Delta m(x)}(\eta)
$$

Therefore, from (3.27) it follows that for every $m \in\{-B, \ldots, B\}^{S}$,

$$
\lim _{n \uparrow \infty}\left(\prod_{x \in V_{n}} a_{x}^{\Delta m(x)}\right)(\eta)=\lim _{n \uparrow \infty}\left(\prod_{x \in \partial V_{n}} a_{x}^{\Delta m(x)}\right)(\eta)=\eta
$$

Therefore, if $\eta, \xi \in \Omega_{\mu}$ are satisfy

$$
\eta+\Delta m=\xi
$$

then, using (3.28) and (3.29):

$$
\eta=\lim _{n \rightarrow \infty} \prod_{x \in V_{n}} a_{x}^{\Delta m(x)} \eta=\xi
$$

which shows the desired property of the set $\Omega_{\mu}$.

From now on we denote by $\mu$ the unique element of $\mathcal{I}$ as well as the Haar measure.

\section{Infinite volume dynamics}

From the previous sections we know that $\mathcal{I}$ contains a unique element $\mu$ and that addition operators as well as their inverses are well-defined on $\mu$-typical configurations. This measure $\mu$ is the natural candidate for a stationary measure of a Markov process on infinite volume recurrent configurations. The construction of this Markov process is completely identical to what was done in [10]. We therefore state the results on existence and Poisson representation of this process without proofs, in the following section, and proceed in section 4.2 to the proof of its ergodic properties, which was open in [10]. 


\subsection{Infinite volume Markov process}

For the unique $\mu \in \mathcal{I}$ we can construct a stationary Markov process on $\mu$-typical infinite volume configurations, as in [10].

We assume that the addition rate function $\varphi$ introduced in (2.4) satisfies

$$
\sup _{y \in S} \sum_{x \in S} \varphi(x) G(y, x)<\infty
$$

This condition ensures that the number of topplings at any site $x \in S$ remains finite almost surely in any finite interval of time when grains are added at intensity $\varphi$. Notice that for dissipative systems, by (2.12), we can take the addition rate function constant.

To each site $x \in S$ we associate a Poisson process $N_{\varphi}^{t, x}$ (for different sites these Poisson processes are mutually independent) with rate $\varphi(x)$. At the event times of $N_{\varphi}^{t, x}$ we "add a grain" at $x$, i.e., we apply the addition operator $a_{x}$ to the configuration. For every finite volume $V \in \mathcal{S}$, the natural extension of (2.4)

$$
L_{V}^{\varphi}=\sum_{x \in V} \varphi(x)\left(a_{x}-I\right)
$$

is the $L^{p}(\mu)$ generator of the stationary pure jump process on $\Omega$ with semigroup

$$
S_{V}^{\varphi}(t)=\exp \left(t L_{V}^{\varphi}\right) f=\int\left(\prod_{x \in V} a_{x}^{N_{\varphi}^{t, x}} f\right) d \mathbb{P},
$$

where $\mathbb{P}$ denotes the joint distribution of the independent Poisson processes $\left\{N_{\varphi}^{t, x}\right\}$, and $f \in L^{p}(\mu)$. The following theorems can be derived directly from the techniques developed in [10].

Theorem 4.4 If $\varphi$ satisfies condition (4.1), then

1. The semigroups $S_{V}^{\varphi}(t)$ converge strongly in $L^{1}(\mu)$ to a semigroup $S_{\varphi}(t)$.

2. $S_{\varphi}(t)$ is the $L^{1}(\mu)$ semigroup of a stationary Markov process $\left\{\eta_{t}: t \geq 0\right\}$ on $\Omega$.

3. For any $f \in \mathcal{L}$,

$$
\lim _{t \downarrow 0} \frac{S_{\varphi}(t) f-f}{t}=L^{\varphi} f=\sum_{x \in S} \varphi(x)\left[a_{x} f-f\right]
$$

where the limit is taken in $L^{1}(\mu)$.

4. The process $\left\{\eta_{t}: t \geq 0\right\}$ admits a càdlàg version (right-continuous with left limits).

The intuitive description of the process $\left\{\eta_{t}: t \geq 0\right\}$ is correct under condition (4.1), i.e., the process has a representation in terms of Poisson processes: 
Theorem 4.5 Assume (4.1). For $\mu \times \mathbb{P}$ almost every $(\eta, \omega)$ the limit

$$
\lim _{V \uparrow S} \prod_{x \in V} a_{x}^{N_{\varphi}^{t, x}(\omega)} \eta=\eta_{t}
$$

exists. The process $\left\{\eta_{t}: t \geq 0\right\}$ is a version of the process of Theorem 4.4, i.e., its $L^{1}(\mu)$ semigroup coincides with $S_{\varphi}(t)$.

To formulate next theorem we need a partial order on configurations, functions, and probability measures on $\Omega$. For $\eta, \xi \in \Omega, \eta \leq \xi$ if $\eta(x) \leq \xi(x)$ for all $x \in S$. A function $f: \Omega \rightarrow \mathbb{R}$ is monotone if $\eta \leq \xi$ implies $f(\eta) \leq f(\xi)$, for all $\eta, \xi \in \Omega$. For two probability measures $\nu, \nu^{\prime}$ on $\Omega, \nu \leq \nu^{\prime}$ if $\nu(f) \leq \nu^{\prime}(f)$ for all monotone bounded Borel measurable function $f$.

Theorem 4.6 Let $\nu \leq \mu$. For $\nu \times \mathbb{P}$ almost every $(\eta, \omega)$ the limit

$$
\lim _{V \uparrow S} \prod_{x \in V} a_{x}^{N_{\varphi}^{t, x}(\omega)} \eta=\eta_{t}
$$

exists. The process $\left\{\eta_{t}: t \geq 0\right\}$ is Markovian with $\eta_{0}$ distributed according to $\nu$.

Remark 4.7 Theorem 4.6 implies that $\eta \equiv 1$ can be taken as initial configuration.

\subsection{An ergodic theorem}

In the rest of this section, we assume for simplicity that the rate function $\varphi \equiv 1$ is constant, and we write $S(t)$ (see Theorem 4.4), $N^{t, x}, L$ and $L_{V}^{0}$ (see (2.4)) without subscript $\varphi$.

We investigate the convergence of $\nu S(t)$ to $\mu$ for a measure $\nu \leq \mu$.

Before we give the statement and its proof, observe that the role of the dissipativity parameter $\gamma$ here is double. First, the approximation (and even the existence) of the infinite volume process by finite volume ones gets nicer and easier to prove when $\gamma$ increases. It is essentially based on the dissipativity condition (2.12). On the other hand, in finite volume, the exponential relaxation to the stationary measure $\mu_{V}$ also depends on $\gamma$ and in fact, becomes slower for larger $\gamma$. This can be seen from ignoring (as would be reasonable for very large $\gamma$ and dimension $d$ ) the interaction with other sites: we then have essentially a one site dynamics by which at exponential times one grain is added to the site until the latter reaches a height $\gamma+1$, after which it topples to height 1 and so on. The relaxation time of this dynamics being clearly proportional to $\gamma$, the convergence is slower for larger $\gamma$.

Theorem 4.8 Suppose $\nu$ is a probability measure on $\Omega$ such that $\nu \leq \mu$. There is a constant $C_{2}>0$ so that for all $f \in \mathcal{L}$, there exist $C_{f}<+\infty$

$$
\left|\int S(t) f d \nu-\int f d \mu\right| \leq C_{f} \exp \left(-C_{2} t\right)
$$

In particular, $\nu S(t)$ converges weakly to $\mu$, uniformly in $\nu \leq \mu$ and exponentially fast. 
Proof. The idea is to approximate $S(t)$ by finite volume semigroups, and to estimate the speed of convergence as a function of the volume. More precisely, we split

$$
\left|\int S(t) f d \nu-\int f d \mu\right| \leq A_{t}^{V}(f)+B_{t}^{V}(f)+C_{V}(f)
$$

with

$$
\begin{aligned}
& A_{t}^{V}(f)=\left|\int S(t) f d \nu-\int S_{V}(t) f d \nu_{V}\right| \\
& B_{t}^{V}(f)=\left|\int S_{V}(t) f d \nu_{V}-\int f d \mu_{V}\right| \\
& C_{V}(f)=\left|\int f d \mu_{V}-\int f d \mu\right| .
\end{aligned}
$$

where $\nu_{V}$ is the restriction of $\nu$ to $V$ and

$$
S_{V}(t) f(\eta)=\int f\left(\prod_{x \in V} a_{x, V}^{N^{t, x} \eta}\right) d \mathbb{P}
$$

By application of Theorem 3.25,

$$
\lim _{V \uparrow S} C_{V}(f)=0
$$

For the first term in the right-hand side of $(4.10)$ we write

$$
A_{t}^{V}(f)=\left|\iint\left(f\left(\prod_{x \in S} a_{x}^{N^{t, x}} \eta\right)-f\left(\prod_{x \in V} a_{x, V}^{N^{t, x}} \eta\right)\right) d \mathbb{P} d \nu\right|
$$

The integrand of the right hand side is zero if no avalanche from $V^{c}$ has influenced sites of $D_{f}$ during the interval $[0, t]$, otherwise it is bounded by $2\|f\|_{\infty}$. Therefore, since $N^{t, x}$ are rate one Poisson processes:

$$
A_{t}^{V}(f) \leq 2\|f\|_{\infty} t \sum_{y \in D_{f}} \sum_{x \in V^{c}} G(x, y)
$$

The second term in the right hand side of (4.10) is estimated by the relaxation to equilibrium of the finite volume dynamics. The generator $L_{V}^{0}$ has the eigenvalues

$$
\sigma\left(L_{V}^{0}\right)=\left\{\sum_{x \in V}\left(\exp \left(2 \pi i \sum_{y \in V} G_{V}(x, y) n_{y}\right)-1\right): n \in \mathbb{Z}^{V} / \Delta^{V} \mathbb{Z}^{V}\right\}
$$

The eigenvalue 0 corresponding to the stationary state arises from the choice $n=\overline{0}$. For the speed of relaxation to equilibrium we are interested in the minimum absolute value of the real part of the non-zero eigenvalues. More precisely:

$$
B_{t}^{V}(f) \leq C_{f} \exp \left(-\lambda_{V} t\right)
$$


where

$$
\begin{aligned}
\lambda_{V} & =\inf \left\{|\operatorname{Re}(\lambda)|: \lambda \in \sigma\left(L_{V}^{0}\right) \backslash\{0\}\right\} \\
& =2 \inf \left\{\sum_{x \in V} \sin ^{2}\left(\pi \sum_{y \in V} G_{V}(x, y) n_{y}\right): n \in \mathbb{Z}^{V} / \Delta^{V} \mathbb{Z}^{V}, n \neq \overline{0}\right\}
\end{aligned}
$$

by (4.14). Since there exists $c>0$ such that for all real numbers $r$

$$
\sin ^{2}(\pi r) \geq c(\min \{|r-k|: k \in \mathbb{Z}\})^{2}
$$

we get

$$
\sum_{x \in V} \sin ^{2}\left(\pi\left(\Delta^{-1} n\right)_{x}\right) \geq \operatorname{cinf}\left\{\left\|\left(\Delta^{V}\right)^{-1} n-k\right\|^{2}: n \in \mathbb{Z}^{V} / \Delta^{V} \mathbb{Z}^{V}, n \neq 0, k \in \mathbb{Z}^{V}\right\}
$$

where $\|\cdot\|$ represents the euclidian norm in $\mathbb{Z}^{V}$ that we estimate by

$$
\left\|\left(\Delta^{V}\right)^{-1} n-k\right\|^{2}=\left\|\left(\Delta^{V}\right)^{-1}\left(n-\Delta^{V} k\right)\right\|^{2} \geq\left\|\Delta^{V}\right\|^{-2}
$$

For any regular volume we have

$$
\left\|\Delta^{V}\right\| \leq \sqrt{2 \gamma^{2}+16 d^{2}}
$$

This gives

$$
B_{t}^{V}(f) \leq C_{f} \exp (-C t)
$$

where $C>0$ is independent of $V$.

The statement of the theorem now follows by combining (4.11), (4.13), (4.16).

Remark 4.17 When we restrict ourselves to the case where

$$
\sum_{x \in S} \varphi(x)=M<\infty
$$

$L^{\varphi}$ becomes a bounded operator, hence it generates a pure jump process which is a continuous time random walk on the group $(\mathcal{R} / \sim, \oplus)$. By the ergodic properties of random walks on compact groups we then obtain that

$$
\lim _{t \rightarrow \infty} \nu S_{\varphi}(t)=\mu
$$

for every measure $\nu$ on $\mathcal{R} / \sim$ (see Theorems 2.5.14, 2.6.2 and Corollary 2.6.4 in [7] for details). 


\subsection{Mixing property}

To the stationary process defined in Theorem 4.4 , we associate the process on $\mathcal{R} / \sim$ by putting

$$
[\eta]_{t}=\left[\eta_{t}\right] .
$$

For that, it is important to notice that the equivalence of recurrent configurations is preserved in time (by Theorem 4.5, and points 1,2 of Remark 3.18): when $\eta \sim \xi$, then $\eta_{t} \sim \xi_{t}$ with $\mathbb{P}$-probability one. For the following theorem, we abbreviate without consequences $[\eta]_{t}=\eta_{t}$.

Theorem 4.20 The process $\left\{\eta_{t}: t \geq 0\right\}$ is mixing, i.e., for all $f, g \in \mathcal{L}$ :

$$
\lim _{t \rightarrow \infty} \int(S(t) f) g d \mu=\int f d \mu \int g d \mu
$$

Proof. Since the semigroup is a normal operator on $L^{2}(\mu)$, ergodicity of the process implies the mixing property by [13]. It thus suffices to prove that for a bounded nonnegative function $f$ such that $\int f d \mu>0$ and $L f=0$, then $\mu$-a.s. $f=\int f d \mu$. By the invariance of $\mu$ under $a_{x}$,

$$
0=-2 \int(f L f) d \mu=\sum_{x \in S} \int\left(a_{x} f-f\right)^{2} d \mu
$$

which implies $a_{x} f=f$ for all $a_{x}, \mu$-a.s. Hence, the measure

$$
d \nu_{f}=\frac{f d \mu}{\int f d \mu}
$$

is invariant under the action of $a_{x}$, thus under the group action. By uniqueness of the Haar measure, we conclude $\nu_{f}=\mu$.

\section{Decay of correlations}

In this section we prove that the infinite volume measure $\mu$ has exponential decay of correlations under a condition of "strong dissipativity". That means for the model (2.1) with $S=\mathbb{Z}^{d}$ that $\gamma$ must be sufficiently large, e.g. $\gamma>13$ for $d=2$; for the strips $S=\mathbb{Z} \times\{1, \ldots, \ell\}$ with finite $\ell$ it always suffices that $\gamma>3$.

In [11] the exponential decay between very special local observables (indicators of socalled weakly allowed clusters) is also obtained in the case where the Green function decays exponentially. However, the technique developed in that paper does not apply to all local functions. 


\subsection{Decoupling argument}

We start with the heuristics of the main ingredient in the proof of exponential decay of correlations. The rest is based on quite general stochastic-geometric methods that are reviewed in [6].

To be specific, suppose that $S=\mathbb{Z}^{2}$ and $\gamma>4$ (in (2.1)). Then, for every volume $V \in \mathcal{S}$

$$
\mu_{V}(\eta(x)=a \mid \eta(z)=c)=\mu_{V \backslash z}(\eta(x)=a)
$$

for all $a, c \in\{1, \ldots, \gamma\}, c>4, x \neq z$ in $V$, because, by the burning algorithm, we can burn away the sites on which we know that the configuration is sufficiently large. Instead of fixing in (5.1) the height value at one site $z$, we could do the same thing on some region $C \subset V$ that does not contain $x$, see Lemma 5.4. On the other hand, if sites $x$ and $y$ are not very close to each other, we can find volumes $\Lambda_{x}, \Lambda_{y} \subset V$ that contain $x$, respectively $y$, that also do not touch (more precisely, that satisfy $\left(\Lambda_{x} \cup \partial \Lambda_{x}\right) \cap \Lambda_{y}=\emptyset$ ). Then, see Lemma 5.7,

$$
\mu_{\Lambda_{x} \cup \Lambda_{y}}(\eta(x)=a, \eta(y)=b)=\mu_{\Lambda_{x}}(\eta(x)=a) \mu_{\Lambda_{y}}(\eta(y)=b)
$$

The combination of (5.1) with (5.2) yields conditional independence of two events that are separated by a region $C$ where the configuration is sufficiently high, see Lemma 5.9.

Definition 5.3 Let $V \in \mathcal{S}, C \subset V, \sigma \in \Omega_{V}$. The subconfiguration $\sigma_{C}$ is $V$-burnable if there exists a bijection $f:\{1, \ldots, n\} \rightarrow C$ such that

$$
\mathcal{N}_{V}(f(1))<\sigma(f(1))
$$

and for every $j=1, \ldots, n-1$,

$$
\mathcal{N}_{V-\{f(1), \ldots, f(j)\}}(f(j+1))<\sigma(f(j+1)) .
$$

As an example, on $\mathbb{Z}^{2}$ with maximal height $\Delta_{x x}=5$, every closed curve along which the heights are at least 4 and containing at least one point with height 5 is burnable.

Lemma 5.4 Let $V=\Lambda \cup C \in \mathcal{S}, \Lambda \cap C=\emptyset$ and fix an arbitrary configuration $\sigma \in \Omega_{V}$ so that $\sigma_{C}$ is $V$-burnable. Put

$$
E_{C}=\left\{\eta \in \Omega_{V}: \eta_{C}=\sigma_{C}\right\}
$$

Then, for all events $A$ that depend only on the configuration in $\Lambda$ (i.e., $A \in \mathcal{F}_{\Lambda}$ ),

$$
\mu_{V}\left(A \mid E_{C}\right)=\mu_{\Lambda}(A)
$$

Proof. By the burning algorithm, $\eta \in \mathcal{R}_{V} \cap E_{C}$ if and only if $\eta_{\Lambda} \in \mathcal{R}_{\Lambda}$ and $\eta_{C}=\sigma_{C}$. Therefore,

$$
\begin{aligned}
\mu_{V}\left(A \mid E_{C}\right) & =\frac{\sum_{\eta \in \mathcal{R}_{V}} I(\eta \in A) I\left(\eta \in E_{C}\right)}{\sum_{\eta \in \mathcal{R}_{V}} I\left(\eta \in E_{C}\right)} \\
& =\frac{\sum_{\eta_{\Lambda} \in \mathcal{R}_{\Lambda}} \sum_{\eta_{C} \in \mathcal{R}_{C}} I(\eta \in A) I\left(\eta \in E_{C}\right)}{\sum_{\eta_{C} \in \mathcal{R}_{C}} I\left(\eta \in E_{C}\right)\left|\mathcal{R}_{\Lambda}\right|} \\
& =\mu_{\Lambda}(A) .
\end{aligned}
$$


Remark 5.6 We do not need to condition on one fixed burnable configuration. The Lemma and its proof above remain unchanged when taking

$$
E_{C}=\left\{\eta \in \Omega_{V}: \eta_{C} \text { is V-burnable }\right\}
$$

the event that we can burn away the sites of $C$ first.

Lemma 5.7 Let $\Lambda_{1}, \Lambda_{2} \in \mathcal{S}$ with

$$
\left(\Lambda_{1} \cup \partial \Lambda_{1}\right) \cap \Lambda_{2}=\emptyset .
$$

For $A \in \mathcal{F}_{\Lambda_{1}}, B \in \mathcal{F}_{\Lambda_{2}}$

$$
\mu_{\Lambda_{1} \cup \Lambda_{2}}(A \cap B)=\mu_{\Lambda_{1} \cup \Lambda_{2}}(A) \mu_{\Lambda_{1} \cup \Lambda_{2}}(B)
$$

Proof. We have $\eta \in \mathcal{R}_{\Lambda_{1} \cup \Lambda_{2}}$ if and only if $\eta_{\Lambda_{1}} \in \mathcal{R}_{\Lambda_{1}}$ and $\eta_{\Lambda_{2}} \in \mathcal{R}_{\Lambda_{2}}$. The rest is writing out expectations as in the proof of Lemma 5.4

We now state the conditional independence.

Lemma 5.9 For $V \in \mathcal{S}$ and $C \subset V$, suppose that $V \backslash C=\Lambda_{1} \cup \Lambda_{2}$ with $\Lambda_{1}, \Lambda_{2}$ satisfying (5.8). Then, for all $A \in \mathcal{F}_{\Lambda_{1}}, B \in \mathcal{F}_{\Lambda_{2}}$

$$
\mu_{V}\left(A \cap B \mid E_{C}\right)=\mu_{V}\left(A \mid E_{C}\right) \mu_{V}\left(B \mid E_{C}\right)
$$

Proof. By Lemma 5.4,

$$
\mu_{V}\left(A \cap B \mid E_{C}\right)=\mu_{V \backslash C}(A \cap B)
$$

and continuing via Lemma 5.7

$$
\mu_{V}\left(A \cap B \mid E_{C}\right)=\mu_{\Lambda_{1} \cup \Lambda_{2}}(A) \mu_{\Lambda_{1} \cup \Lambda_{2}}(B) .
$$

The proof is finished by applying again Lemma 5.4 to the two factors in the right-hand side of $(5.10)$.

The conditional independence (5.10) is reminiscent of the situation for Markov random fields. Here $\mu_{V}$ is not Markovian but nevertheless for all $A \in \mathcal{F}_{\Lambda}, \Lambda \subset V$ the conditional probability of $A$ given the configuration in $V \backslash \Lambda$ is

$$
\mu_{V}\left(A \mid \eta_{\eta \backslash \Lambda}\right)=\mu_{\Lambda}(A)
$$

whenever $\eta_{\partial \Lambda \cap V}$ is $V$-burnable. In particular, this conditional probability (5.11) is then independent of the particular $\eta_{V \backslash \Lambda}$. 


\subsection{Geometric argument}

From the previous decoupling argument, it is clear how to proceed for the proof of decay of correlations. What needs to be established is that there will be typically some "circuit" $C$, separating two far away dependence sets, where the configuration is burnable. We thus basically end up with a stochastic-geometric or percolation-like argument as also reviewed in [6]. The first thing to see is that burnability is sufficiently probable. We do that first for the strip in Lemma 5.12 and then for the full lattice in Lemma 5.16.

Lemma 5.12 Let $V=\left\{(x, y) \in \mathbb{Z}^{2}:|x| \leq k, y=1, \ldots \ell\right\}$ and $\gamma \geq 4$ in (2.1). Fix some $x_{1},\left|x_{1}\right| \leq k$ and let $C=\left\{\left(x_{1}, y\right) \in V: y=1, \ldots \ell\right\}$. There is $p=p(\ell, \gamma)>0$ (uniformly in $k$ ) such that for all events $E\left(x_{1}\right)$ that only depend on the heights $\eta(x, y)$ with $(x, y) \notin C$,

$$
\mu_{V}\left(\eta(x, y) \geq 4 \text { for all }(x, y) \in C \mid E\left(x_{1}\right)\right) \geq p>0
$$

Proof. Via Bayes'rule,

$$
\begin{array}{ll}
\mu_{V}\left(\eta(x, y) \geq 4 \text { for all }(x, y) \in C \mid E\left(x_{1}\right)\right) & = \\
\mu_{V}\left(E\left(x_{1}\right) \mid \eta(x, y) \geq 4 \text { for all }(x, y) \in C\right) & \frac{\mu_{V}(\eta(x, y) \geq 4 \text { for all }(x, y) \in C)}{\mu_{V}\left(E\left(x_{1}\right)\right)}
\end{array}
$$

If $\eta(x, y) \geq 4$ for the points $(x, y) \in C$, then $\eta_{C}$ is $V$-burnable, and by Lemma 5.4

$$
\mu_{V}\left(E\left(x_{1}\right) \mid \eta\left(x_{1}, y_{1}\right) \geq 4 \text { whenever }\left|y_{1}\right| \leq \ell\right)=\mu_{V \backslash C}\left(E\left(x_{1}\right)\right)
$$

On the other hand, by counting,

$$
\frac{\mu_{V \backslash C}\left(E\left(x_{1}\right)\right)}{\mu_{V}\left(E\left(x_{1}\right)\right)} \geq \frac{\left|\mathcal{R}_{V}\right|}{\left|\mathcal{R}_{V \backslash C}\right|\left|\mathcal{R}_{C}\right|}
$$

and

$$
\mu_{V}(\eta(x, y) \geq 4 \text { for all }(x, y) \in C)=(\gamma-4+1)^{\ell} \frac{\left|\mathcal{R}_{V \backslash C}\right|}{\left|\mathcal{R}_{V}\right|}
$$

As a consequence we can take

$$
0<p \leq \frac{(\gamma-4+1)^{\ell}}{\left|\mathcal{R}_{C}\right|}
$$

Remark 5.15 Obviously, $p \downarrow 0$ as $\ell \uparrow \infty$.

For the regular lattice $S=\mathbb{Z}^{d}$ we have: 
Lemma 5.16 Consider the model (2.1) with $S=\mathbb{Z}^{d}$. For all $\varepsilon>0$, there is a $\gamma_{0}<+\infty$ so that for all $V \in \mathcal{S}$, all $x \in V$ and all events $E \in \mathcal{F}_{V \backslash x}$,

$$
\mu_{V}(\eta(x)>2 d \mid E)>1-\varepsilon
$$

whenever $\gamma \geq \gamma_{0}$.

Proof. We can repeat the steps of the proof in Lemma 5.12. At the end we must estimate the number of burnable heights at $x$ divided by the number of configurations at $x$. That is,

$$
\mu_{V}(\eta(x)>2 d \mid E)>\frac{\gamma-2 d}{\gamma}
$$

It thus suffices that $2 d<\gamma \varepsilon$.

We need one more lemma before giving the geometric argument, because the latter requires stochastic domination by Bernoulli measure.

Lemma 5.17 The invariant probability measure $\mu_{V}$ for the sandpile dynamics in $V$ is irreducible, i.e., for two given recurrent configurations $\eta, \eta^{\prime}$, there is a sequence $\eta_{0}=$ $\eta, \ldots, \eta_{m}=\eta^{\prime}$ of recurrent configurations such that $\eta_{i}$ and $\eta_{i+1}$ differ only in one site.

Proof. Since $\eta^{\prime}$ can be reached from $\eta$ by a finite number of additions, it is enough to show that for any $x \in V$, there is such a sequence from $\eta$ to $a_{x} \eta$. Let

$$
\begin{aligned}
& \Gamma_{x}^{+}(\eta)=\left\{y \in V ;\left(a_{x} \eta\right)(y)>\eta(y)\right\}, \\
& \Gamma_{x}^{-}(\eta)=\left\{y \in V ;\left(a_{x} \eta\right)(y)<\eta(y)\right\} .
\end{aligned}
$$

We first add sand grains one by one on the sites $y \in \Gamma_{x}^{+}(\eta)$, to reach the value $\left(a_{x} \eta\right)(y)$. Each step leads to a configuration larger than $\eta$, thus recurrent. We denote by $\eta^{+}=\eta_{0}^{+}$ the recurrent configuration $\left(a_{x} \eta\right)_{\Gamma_{x}^{+}(\eta)} \eta_{\left[\Gamma_{x}^{+}(\eta)\right]^{c}}$. If $\Gamma_{x}^{-}(\eta)=\emptyset$ we are finished. If not, we write $\Gamma_{x}^{-}(\eta)=\left\{z_{1}, \ldots, z_{n}\right\}$ and we pass from $\eta_{k}^{+}=\left(a_{x} \eta\right)_{\left\{z_{1}, \ldots, z_{k}\right\}} \eta_{\left\{z_{1}, \ldots, z_{k}\right\}^{c}}^{+}$to $\eta_{k+1}^{+}$ for every $1 \leq k \leq n\left(\eta_{k}^{+} \geq a_{x} \eta\right.$ is recurrent and differs from $\eta_{k-1}^{+}$in one site) to reach $\eta_{n}^{+}=a_{x} \eta$.

We are now in a position to give the main stochastic-geometric argument leading to exponential decay of correlations. It copies the proof that complete analyticity for Markovian random fields follows from absence of disagreement percolation, as done in [2], see Theorem 7.1 in [6], except that we can replace the Markov property by the decoupling property (5.11).

Let $p_{c}(d)$ denote the percolation threshold for Bernoulli site percolation on $\mathbb{Z}^{d}$. Let $V \in \mathcal{S}$ and let $\Lambda \subset V$ on which we fix two arbitrary height configurations $\eta_{\Lambda}$ and $\eta_{\Lambda}^{\prime}$ to consider two conditional probabilities $\mu_{1}=\mu_{V}\left(\cdot \mid \eta_{\Lambda}\right)$ and $\mu_{2}=\mu_{V}\left(\cdot \mid \eta_{\Lambda}^{\prime}\right)$.

Theorem 5.18 Suppose that $\gamma \geq 4$ for $S=\mathbb{Z} \times\{1, \ldots, \ell\}$ or $4 d<\gamma p_{c}(d)$ for $S=\mathbb{Z}^{d}$ in (2.1). There exist constants $\alpha>0, C<+\infty$ so that for all $V \in \mathcal{S}, \Lambda \subset V, W \subset$ $V \backslash \Lambda, \eta \in \mathcal{R}$ and for every event $A \in \mathcal{F}_{W}$,

$$
\left|\mu_{1}(A)-\mu_{2}(A)\right| \leq C e^{-\alpha d i s t(W, \Lambda)}
$$

where $\operatorname{dist}(\cdot, \cdot)$ is the nearest neighbor distance between the two subsets. 
Proof. We give the proof for the lattice $S=\mathbb{Z}^{d}$. The case of the strip is analogous but a little simpler (using Lemma 5.12).

We use a coupling argument. First, we introduce some linear ordering on $V \subset \Lambda$. We construct via iteration a coupling between $\mu_{1}$ and $\mu_{2}$ which is a random field $\left(X, X^{\prime}\right)$. We start by setting $X(x)=\eta(x), X^{\prime}(x)=\eta^{\prime}(x)$ on $\Lambda$. Suppose that we have already realized the coupling as $\left(X, X^{\prime}\right)=\left(\eta, \eta^{\prime}\right)$ on all sites outside some non-empty set $T \subset V \backslash \Lambda$. Consider then the conditional distributions $\mu_{V}\left(\cdot \mid \eta_{V \backslash T}\right)$ and $\mu_{V}\left(\cdot \mid \eta_{V \backslash T}^{\prime}\right)$. One possibility is that on the external boundary both $\eta_{\partial T}$ and $\eta_{\partial T}^{\prime}$ are $V$-burnable. But then, via (5.11), these two conditional probabilities are equal on $T$ and we can take the optimal coupling for which $X=X^{\prime}$ on $T$. Alternatively, we choose the smallest site $x \in T$ having a nearest neighbor $y \in V \backslash T$, for which $X(y) \leq 2 d$ or $X^{\prime}(y) \leq 2 d$ and we find the value $\left(\eta(x), \eta^{\prime}(x)\right)$ for the coupling at $x$ from sampling the optimal coupling between the single site distributions $\mu_{V}\left(X(x)=\cdot \mid \eta_{V \backslash T}\right)$ and $\mu_{V}\left(X^{\prime}(x)=\cdot \mid \eta_{V \backslash T}^{\prime}\right)$. At this step the coupling is defined outside $T \backslash x$ and we can repeat the iteration giving us a coupling between $\mu_{1}$ and $\mu_{2}$.

From the above construction, it is possible that in the coupling $X(x) \neq X^{\prime}(x)$ at some $x \in W$, only if there is a nearest neighbor path from $x$ to $\Lambda$ on which $X(y) \leq 2 d$ or $X^{\prime}(y) \leq 2 d$. On the other hand, no matter what we fix off $y$,

$$
P\left(X(y) \leq 2 d \text { or } X^{\prime}(y) \leq 2 d \mid \eta(z), \eta^{\prime}(z), z \in V \backslash y\right) \leq 2\left(1-\mu_{V}(\eta(y)>2 d \mid \eta(z), z \neq x)\right)
$$

For $\gamma$ large enough (from Lemma 5.16), this is bounded by $p_{c}(d)$. The proof is then concluded via an application of stochastic domination with the Bernoulli product measure (thanks to Lemma 5.17, see Theorem 4.8 in [6]) and using that the cluster-diameter in sub-critical Bernoulli site percolation has an exponential tail.

\section{Examples.}

1. The dissipative system in dimension 2: we have $p_{c}^{*}(2)=0.5927$ (as numerical result). Thus we need to take $\gamma>13$ so that $8<\gamma p_{c}^{*}(2)$.

2. The dissipative system in high dimension. Since $p_{c}^{*}(d) \simeq \frac{1}{2 d}$ for large $d$, we conclude exponential decay of correlations as soon as $\gamma>8 d^{2}$.

\section{References}

[1] Bak, P., Tang, K. and Wiesenfeld, K., Self-Organized Criticality, Phys. Rev. A 38, 364-374 (1988).

[2] van den Berg, J. and Maes, C., Disagreement percolation in the study of Markov fields, Ann. Probab. 25, 1316-1333 (1994).

[3] Daerden, F., Vanderzande, C., Dissipative abelian sandpiles and random walks, Phys. Rev E 63 (2001).

[4] Dhar, D., Self Organised Critical State of Sandpile Automaton Models, Phys. Rev. Lett. 64, No.14, 1613-1616 (1990). 
[5] Dhar, D., The Abelian Sandpiles and Related Models, Physica A 263, 4-25 (1999).

[6] Georgii, H.-O., Häggström, O., Maes, C., The random geometry of equilibrium phases, Phase Transitions and Critical Phenomena, Vol. 18, Eds C. Domb and J.L. Lebowitz (Academic Press, London), 1-142 (2001).

[7] Heyer, H, Probability measures on locally compact groups, Springer, 1977.

[8] E.V. Ivashkevich, Priezzhev, V.B., Introduction to the sandpile model, Physica A 254, 97-116 (1998).

[9] Maes, C., Redig, F., Saada E. and Van Moffaert, A., On the thermodynamic limit for a one-dimensional sandpile process, Markov Proc. Rel. Fields, 6, 1-22 (2000).

[10] Maes, C., Redig, F., Saada E., The abelian sandpile model on an infinite tree, (preprint 2000) to appear in Ann. Probab. (2002).

[11] Mahieu, S., Ruelle, P., Scaling fields in the two-dimensional abelian sandpile model, Preprint (2001).

[12] Meester, R., Redig, F. and Znamenski, D., The abelian sandpile; a mathematical introduction, Markov Proc. Rel. Fields, 7, 509-523 (2002).

[13] Rosenblatt, M., Transition probability operators, Proc. Fifth Berkeley Symposium, Math. Statist. Prob., 2, 473-483 (1967).

[14] Speer, E., Asymmetric Abelian Sandpile Models, J. Stat. Phys. 71, 61-74 (1993).

[15] Tsuchiya, V.T and Katori, M., Phys. Rev E 61, 1183 (2000). 